\title{
Study of the Improved Location Algorithm Based on Chan and Taylor
}

\author{
Liu En-Hua*, Xu Ke-Ming and Jiang Yu
}

Jiangsu College of Information Technology, Wuxi, China

\begin{abstract}
Combining the advantages of Chan algorithm and Taylor series expansion method, the article has proposed an improved localization algorithm in the LTE positioning system. The algorithm eliminates much deviation of the positioning results in Chan algorithm, offers the optimized result as the initial value of Taylor series expansion method, then get the weighted sum of the positioning of the results of the two kinds of algorithm, finally put the final result as the location of the mobile station coordinates. Experimental results show that the proposed algorithm can effectively improve the positioning precision in the noise environment.
\end{abstract}

Keywords: Chan algorithm, Taylor series method, Improved location algorithm, Positioning accuracy, Position Coordinates.

\section{INTRODUCTION}

Chan algorithm [1] is one kind of location algorithm which has analytical solutions, and solves the location coordinates of mobile stations on the basis of least square method (LS) or weighted least square method (WLS). This algorithm uses non-recursive method to conduct resolution on hyperbolic equations. The basic concept of Chan algorithm is: firstly, one intermediate viable is used to convert nonlinear equation into linear equation, and the location coordinates of mobile stations are rough estimated by using weighted least square method, then the location coordinates and other constraint conditions are rough estimated, the location coordinates of mobile stations are precisely estimated by using weighted least square method again and the ultimate coordinate could be obtained.

Taylor series expansion method [2] is one kind of recursive algorithm. Its basic concept is: firstly, one initial estimates location value of mobile station is needed, then Taylor series expansion is conducted on this location, the nonlinear equation could be converted into linear equation by ignoring second order and above second order terms, then least square method is used to estimate, each obtained results by recursion is used in location correction of mobile stations to improve the precision of estimate, finally the estimated location could approach the real location of mobile stations is different Compared with Chan algorithm, Taylor series expansion method has no analytical solution and its operation is relatively complicated. Besides, the initial estimated location value of mobile station could not deviate from the real location excessively, otherwise Taylor series expansion will not convergence.

\section{MODIFIED LOCATION ALGORITHM BASED ON CHAN AND TAYLOR}

The classic Chan algorithm could attain all TDOA by measuring, and obtain a specific analytical solution, however, the performance would be affected in NLOS environment. Taylor series expansion method could conduct recursive operation on the initial estimated location value of mobile station and continuously correct the estimated location value of mobile station to make it approach the real location value, however, this algorithm would significantly affected by the initial location, and the operation process is rather complicated. Therefore, we can explore some location algorithm to obtain one relatively precise initial location at first, then the obtained initial location can be used to conduct resolution with Taylor series expansion method and finally the location result which is more precise will be received.

The literature $[3,4]$ presented a location algorithm which was based on Fang algorithm and Taylor series expansion, it eliminated the location estimation which had eliminated the estimated location value which deviated excessively to improve the precision of location. The literature proposed another synergy location algorithm, it conducted weighted calculation of the results attained by different location algorithm, and the estimated value which had been treated was used as the location coordinates of mobile station. According to the thoughts in literature, we present a modified location algorithm which is based on Chan algorithm and Taylor series expansion.

Assumed that the base station of community where the mobile station is located is noted as No.1, and meanwhile it act as the serving base station for the location system. If the measured TOA of $\mathrm{N}_{\mathrm{BS}}$ base stations has been attained, these TOA values are subtracted from the measured TOA values of serving base station and $\left(\mathrm{N}_{\mathrm{BS}}-1\right)$ TDOA values would be got. If data of the involved $m(m \leq n-1)$ base stations is always used during each location, m TDOA values can be randomly 
chosen from these $\left(\mathrm{N}_{\mathrm{BS}}-1\right)$ ones to become one group and it has $C_{N_{B S-1}}^{m}$ kinds of possibilities.

Chan algorithm is used to obtain the initial estimated location value of mobile station under $C_{N_{B S-1}}^{m}$ kinds of conditions respectively. It is noted as $\left(\hat{x}_{k}, \hat{y}_{k}\right)$, besides, $\mathrm{k}=1$, $2, \cdots, C_{N_{B S-1}}^{m}$. With the increasing distance between mobile station and base station error of the estimated $\left(\hat{x}_{k}, \hat{y}_{k}\right)$ also increase, which would significantly affect the ultimate estimated location result of mobile station, therefore, the initial estimated values which deviates excessively should be eliminated according to some disciplines. $\left(\hat{x}_{k}, \hat{y}_{k}\right)$ should be eliminated when the following condition was satisfied :

$\left|\sqrt{x_{1}-\hat{x}_{k}^{2} \mathrm{C}+\left(y_{1}-\hat{y}_{k}\right)^{2}}-R_{1}\right| \leq R_{\text {threshold }}$

Where, $R_{\text {threshold }}$ is one threshold, which could be alternative to control the error of $\left(\hat{x}_{k}, \hat{y}_{k}\right)$ as required.

Assumed that estimated values of $\eta$ initial coordinates remained after eliminating the values which deviates excessively, the values of the $\eta$ coordinates are averaged and got:

$\left[\begin{array}{l}x_{0} \\ y_{0}\end{array}\right]=\left[\begin{array}{l}\frac{1}{\eta}\left(\sum_{k=1}^{\eta} \hat{x}_{k}\right) \\ \frac{1}{\eta}\left(\sum_{k=1}^{\eta} \hat{y}_{k}\right)\end{array}\right]$

$\left(x_{0}, y_{0}\right)$ is the initial estimated location value of mobile stations which is attained with Chan algorithm. $\left(x_{0}, y_{0}\right)$ is substituted into Taylor series expansion method to conduct recursive resolution and one location result could be attained.

The location results attain with Chan algorithm and Taylor series expansion method should be weighted (Fig. 1). The residual between the measured value and location result is defined in the literature [4]:

$R_{e s}=\sum_{i=1}^{N_{B S}}\left(R_{i}-\hat{R}_{i}\right)^{2}$

Where, $\quad \hat{R}_{i}=\sqrt{\left(\hat{x}-x_{i}\right)^{2}+\left(\hat{y}-y_{i}\right)^{2}}, \quad(\hat{x}, \hat{y})$ is the obtained location coordinates of mobile station with location algorithm, $\left(x_{k}, y_{k}\right)$ is the coordinates of No.i base station involved in location, $R_{i}$ is the obtained distance between the mobile station and No.i base station according to TOA value, $N_{B S}$ is the amount of base stations involved in location. When some location algorithm is adopted, if $R_{e s}$ is relatively small, it demonstrates that the obtained coordinate location of the mobile station using this algorithm is more precise; if $R_{e s}$ is relatively large, it indicates that errors of the obtained results using this algorithm is relatively large. The algorithm which is more precise should make up larger proportion in ultimate weighting. The calculation formula of weighing coefficient $R_{k}$ is given in literature [5]. If $\mathrm{k}$ kinds of location algorithms are used to conduct operation on the same group of TDOA, respectively, weighting coefficient $R_{k}$ of No.k location algorithm could be expressed as:

$$
\begin{aligned}
R_{k} & =\frac{R_{e s}^{k}}{N_{B S}}=\frac{1}{N_{B S}} \sum_{i=1}^{N_{B S}}\left(R_{i}-\hat{R}_{i}^{k}\right)^{2} \\
& =\frac{1}{N_{B S}} \sum_{i=1}^{N_{B S}}\left(R_{i}-\sqrt{\left(\hat{x}^{k}-x_{i}\right)^{2}+\left(\hat{y}^{k}-y_{i}\right)^{2}}\right)^{2}
\end{aligned}
$$

besides, $\left(\hat{x}^{k}, \hat{y}^{k}\right)$ is obtained location result by using No.k location algorithm, so the ultimate location coordinate of mobile station $(\hat{x}, \hat{y})$ is:

$$
\left[\begin{array}{l}
\hat{x} \\
\hat{y}
\end{array}\right]=\left[\begin{array}{l}
\frac{1}{\sum_{k=1}^{K} R_{k}^{-1}}\left(\sum_{k=1}^{K} \hat{x}^{k} R_{k}^{-1}\right) \\
\frac{1}{\sum_{k=1}^{K} R_{k}^{-1}}\left(\sum_{k=1}^{K} \hat{y}^{k} R_{k}^{-1}\right)
\end{array}\right]
$$

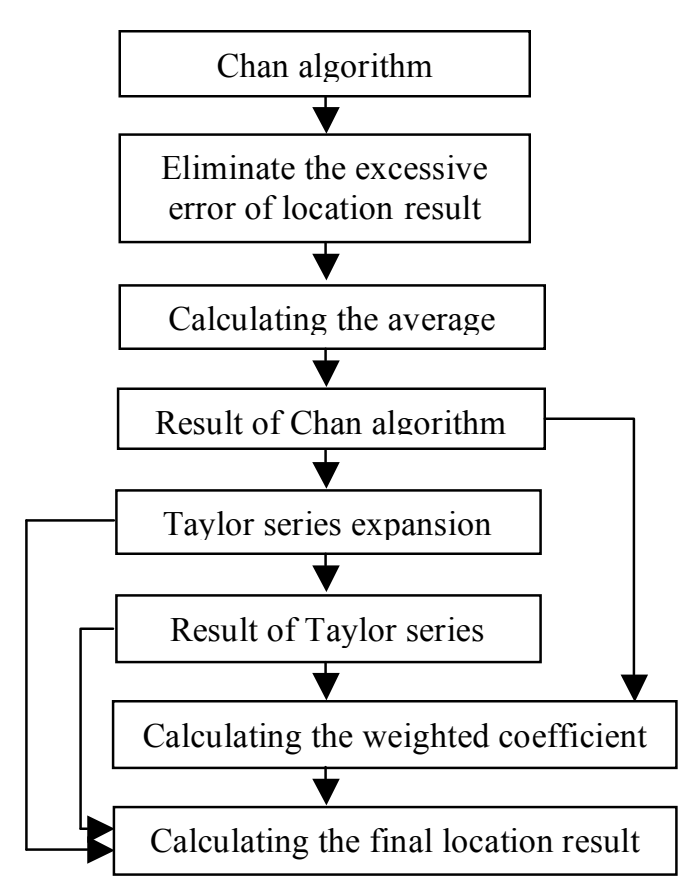

Fig. (1). Flow chart of modified algorithm based on chan and taylor.

\section{SIMULATION AND ANALYSIS}

Simulation is carried out independently for 1000 times on Chan algorithm, Taylor series expansion method and the modified location algorithm which is based on Chan and Taylor, respectively. The model of multiple base station is used in simulation. Assumed that the location system contains seven base stations, and the community radius is 1000 meters, schematic diagram of distribution of base stations are shown in (Fig. 2).

The measured TDOA [6] values which is obtained by using five base stations and using six base stations are simulated, respectively, location of mobile station is A or B. 
The model of signal transmission distance could be expressed:

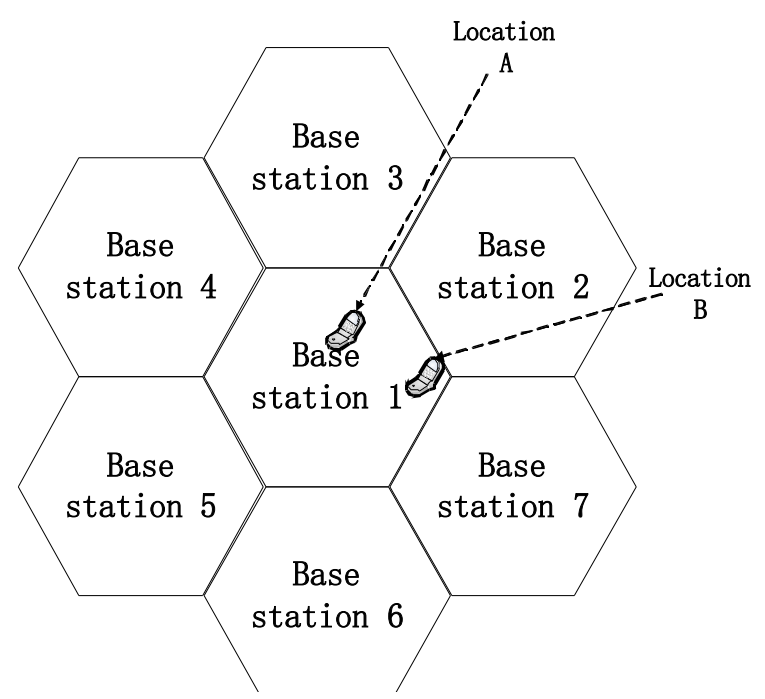

Fig. (2). Schematic diagram of distribution of base stations.

$r_{i}=R_{i}+C_{\text {Gauss }} \times \operatorname{randn}(1)+C_{N L O S} \times \operatorname{rand}(1)$

where, $R_{i}$ is the real distance between mobile station and base station, $\operatorname{randn}(1)$ is one random number between 0 and $1, C_{\text {Gauss }}$ is the noise coefficient which satisfies the normal distribution, $\operatorname{rand}(1)$ is one random number between 0 and 1 which satisfies uniform distribution, $\mathrm{C}_{\mathrm{NLOS}}$ is NLOS coefficient.

Fig. (3) shows the RMSE graph with changing the noise coefficient $[7,8]$ when mobile station is at location A. We can find that the errors of three algorithms all increased with the increase of noise coefficient. The real coordinates of mobile station is adopted as the initial value during simulating the Taylor series expansion method in noise environment, therefore, the precision of Taylor series expansion method is significantly higher than Chan algorithm. The modified algorithm presented in this paper can make the initial value which is used to substitute into Taylor series expansion method approach to the real coordinate values of mobile station for it eliminates the estimated values which are excessively deviated after using Chan algorithm, therefore, the error of the modified algorithm is smaller than Chan algorithm [9, 10]. Due to some deviations existing between the initial values adopted in modified algorithm and real values, its errors are larger than that of Taylor series expansion. When the mobile station is located in location $\mathrm{A}$, the increasing amount of base station make the results of Chan algorithm more precise, however, it has little effects on the error of Taylor series expansion. To the modified algorithm which is based on Chan and Taylor, for the increasing amount of base station decreases the error of Chan algorithm, and increases the precision of initial values to some extent, thus making the results of Taylor series expansion method more precise, the errors of weighted results became smaller.

Fig. (4) shows the RMSE graph with changing the noise coefficient when the mobile station is at location B. From the graph it could be seen that location variation has some effects on location precision. When the mobile station is at location $\mathrm{B}$, the increasing amount of base stations also improve the location precision of Taylor series expansion method. From (Figs. 3 and 4), we can find that in noise environment, the proposed algorithm attains better performance than Chan algorithm at different locations.

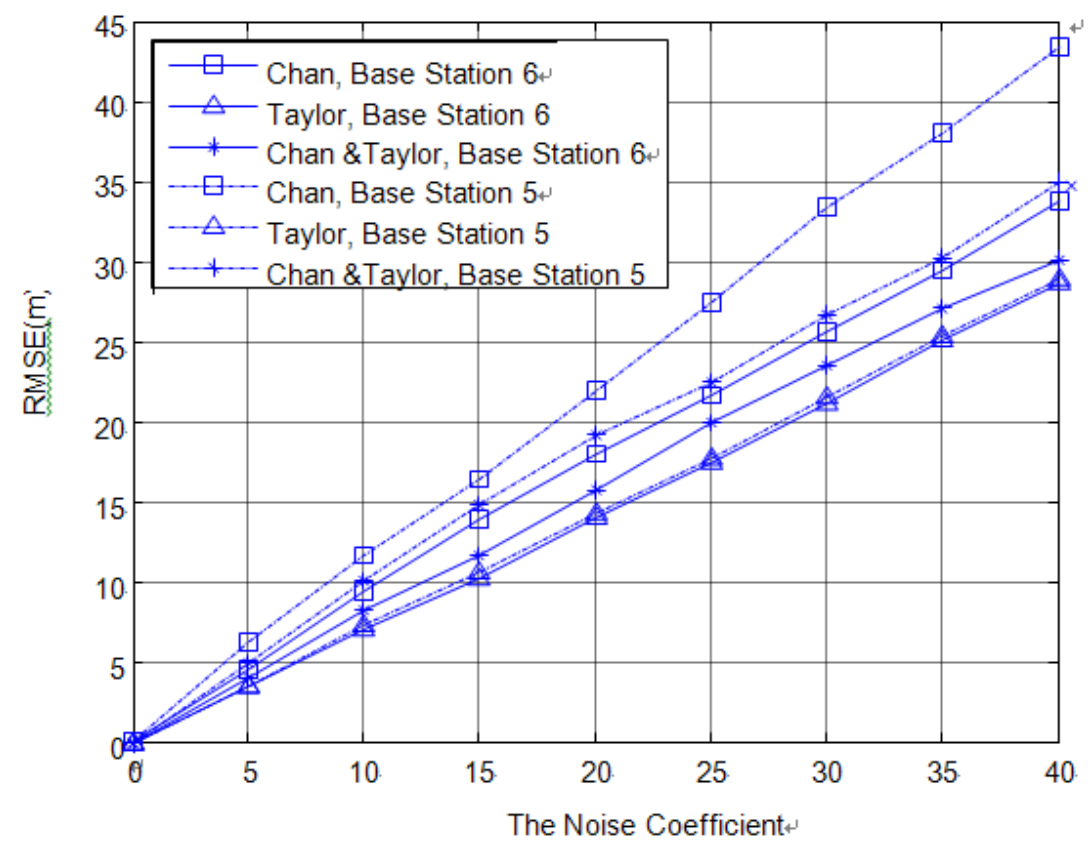

Fig. (3). RMSE graph with changing the noise coefficient when mobile station is at location A. 


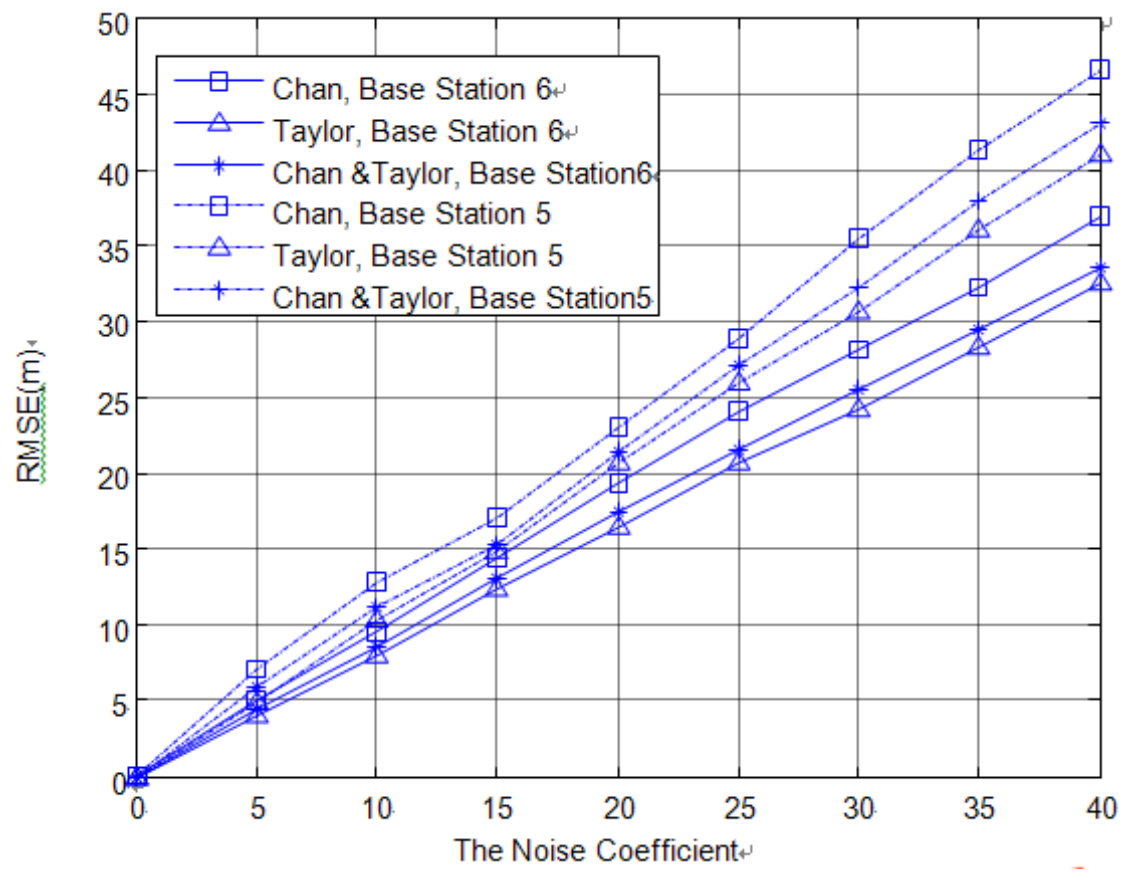

Fig. (4). RMSE graph with changing the noise coefficient when mobile station is at location B.

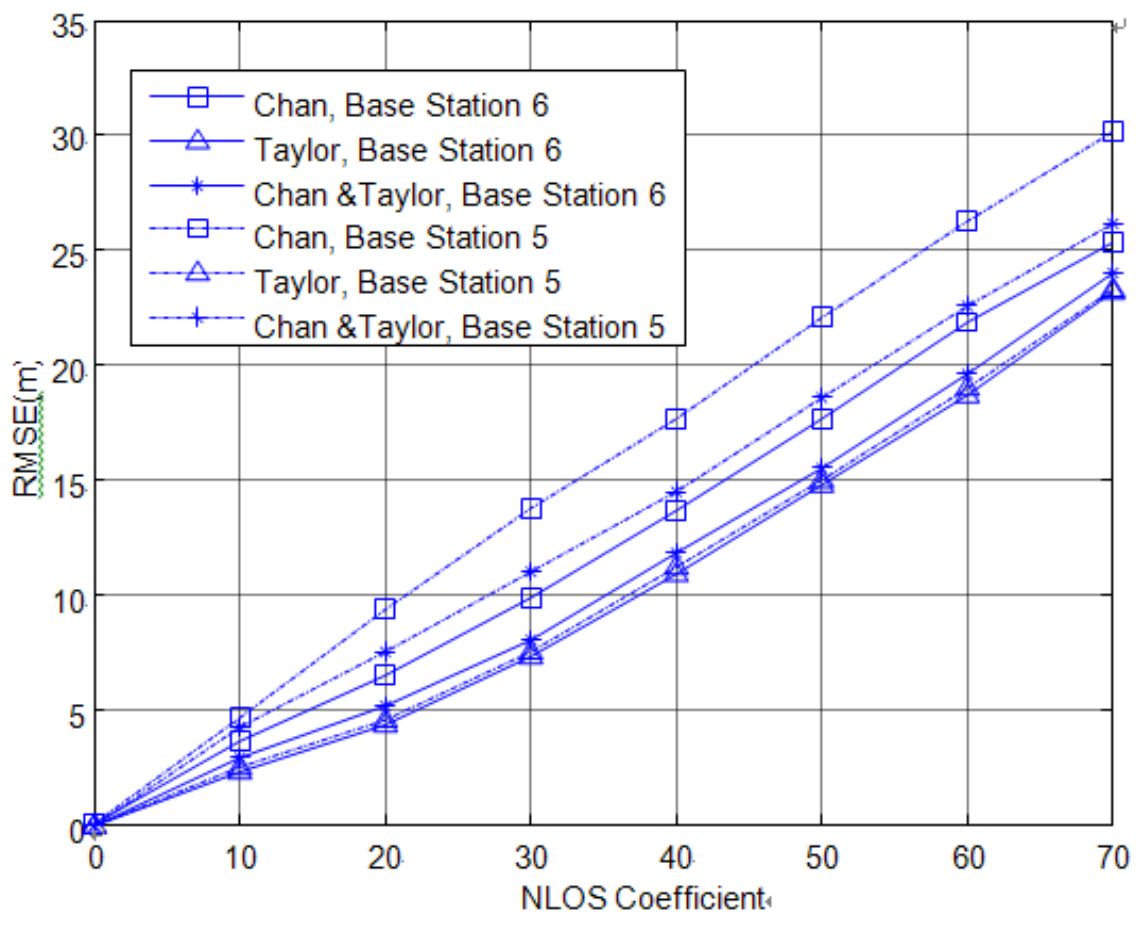

Fig. (5). RMSE graph with changing nlos coefficient when mobile station is at location A.

Figs. (5 and 6) show the RMSE graphs with changing NLOS coefficient when mobile stations are at location A and $\mathrm{B}$, respectively. From these graphs we find that with the increase of NLOS coefficient, performance of the three algorithms all decrease. In NLOS environment, the location precision of Taylor series expansion method is obviously better than Chan algorithm, and the location variation of mobile station also effects on location results. Because of integrating the advantages of Chan algorithm and Taylor series expansion method, the performance of our proposed algorithm is also better than Chan algorithm in NLOS environment.

\section{CONCLUSION}

In this article, we present a modified location algorithm which is based on Chan and Taylor. Firstly, this algorithm uses Chan algorithm to estimate the location of mobile 


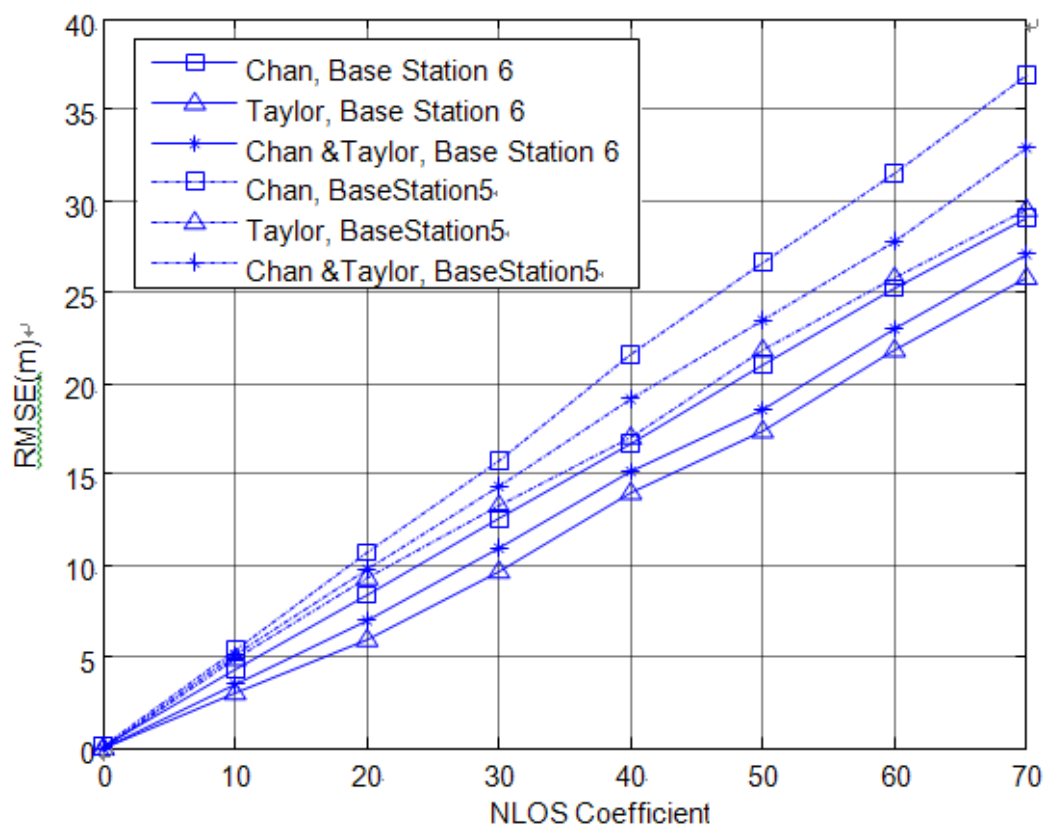

Fig. (6). RMSE graph with changing nlos coefficient when mobile station is at location B.

station, then the obtained results are optimized by eliminating the contained location results which deviated excessively, the obtained optimized results are acted as the initial value used in Taylor series expansion method to conduct another location operation, finally, the obtained location results using these two algorithms are weighted summed, and the ultimate result is the location coordinate of mobile station. The experiment indicates that in the noise environment, the performance of the proposed algorithm in the article is better than Chan algorithm, which could effectively improve the location precision.

\section{CONFLICT OF INTEREST}

The authors confirm that this article content has no conflict of interest.

\section{ACKNOWLEDGEMENTS}

Declared none.

\section{REFERENCES}

[1] T. Yang, L. Jin, and J. Cheng, "An improvement CHAN algorithm based on TOA position," Acta Electronica Sinica, vol. 4, p. 026, 2009.

[2] W. H. Foy, "Position-location solutions by Taylor-series estimation," IEEE Transactions on Aerospace and Electronic Systems, pp. 187-194, 1976.
[3] M. C-Kun, C. S-Xin , W. Hao, and Z. Yi-Hang, "Recursion leastsquares passive location algorithm based on angle information", Computer Measurement \& Control, vol. 22, no.9, pp. 2863-2866, 2004.

[4] W. Cui, C. Wu, Y. Zhang, Z.-x. Jia, and L. Cheng, "GMM-based localization algorithm under NLOS conditions," Journal on Communications, vol. 35, pp. 99-106, 2014.

[5] G. F-Xiang, W. Qing, and Z. Xiao-Guo,"TDOA/AOA hybrid wireless location algorithm in cellular networks with NLOS condition," Journal of Southeast University, vol.9, pp. 905-910, 2010.

[6] G. Fuxiang, W. Qing, and Z. Xiaoguo, "Hybrid GPS/TDOA steepest decent location algorithm in non-line-of-sight environment," Chinese Journal of Scientific Instrument, vol. 7, p. $009,2011$.

[7] T. J. Khanzada, A. R. Ali, and A. S. Omar, "Time difference of arrival estimation using super resolution algorithms to minimize distance measurement error for indoor positioning systems," In: IEEE International Multitopic Conference, INMIC, 2008, pp. 443447.

[8] P.-C. Chen, "A non-line-of-sight error mitigation algorithm in location estimation," In: Wireless Communications and Networking Conference, IEEE, 1999, pp. 316-320.

[9] P. J. Voltz and D. Hernandez, "Maximum likelihood time of arrival estimation for real-time physical location tracking of $802.11 \mathrm{a} / \mathrm{g}$ mobile stations in indoor environments," In: Position Location and Navigation Symposium, 2004, pp. 585-591.

[10] Y. Chan and K. Ho, "A simple and efficient estimator for hyperbolic location," IEEE Transactions on Signal Processing, vol. 42, pp. 1905-1915, 1994.

(C) En-Hua et al.; Licensee Bentham Open.

This is an open access article licensed under the terms of the (https://creativecommons.org/licenses/by/4.0/legalcode), which permits unrestricted, noncommercial use, distribution and reproduction in any medium, provided the work is properly cited. 